\title{
POLY-SIGE: A HIGH- $Q$ STRUCTURAL MATERIAL FOR INTEGRATED RF MEMS
}

\author{
Sunil A. Bhave ${ }^{1}$, Brian L. Bircumshaw ${ }^{2}$, Wing Zin Low ${ }^{1}$, Yong-Sang Kim ${ }^{1, \dagger}$, Albert P. Pisano ${ }^{2,1}$, \\ Tsu-Jae King ${ }^{1}$, and Roger T. Howe ${ }^{1,2}$ \\ Department of Electrical Engineering \& Computer Sciences ${ }^{1}$ and Department of Mechanical Engineering ${ }^{2}$ \\ Berkeley Sensor \& Actuator Center, 497 Cory Hall, University of California, Berkeley, CA 94720-1774
}

\begin{abstract}
This paper presents new material data for single- and multilayer CMOS compatible poly-SiGe films, including mechanical quality factor $(Q)$, Young's modulus, and strain gradient. Using audio frequency folded-flexure comb-drive resonators, the mechanical quality factor of poly-SiGe was determined at $2 \mu \mathrm{Torr}$ pressure. As-deposited poly-SiGe has $Q=20,000-31,000$. CMOS compatible rapid thermal annealing (RTA) at $525^{\circ} \mathrm{C}$ for 60 seconds results in a quality factor between 40,000 and 44,000 . We have measured the highest $Q$ factor yet reported for poly-SiGe $(Q \approx 61,100)$, as a result of RTA at $600^{\circ} \mathrm{C}$ for one minute. The measured resonant frequencies of the resonators were used to back-calculate the Young's modulus of poly-SiGe: $E=155 \pm 5 \mathrm{GPa}$. The calculated elastic modulus is significantly higher than the metallurgical Young's modulus of 146GPa. The as-deposited strain gradient of the tri-layer poly-SiGe film was found to be $1.75 \cdot 10^{-4} \mu \mathrm{m}^{-1}$ (curl-up). RTA at $600^{\circ} \mathrm{C}$ for one minute drops the tri-layer strain gradient to $2.67 \cdot 10^{-5} \mu \mathrm{m}^{-1}$ (curl-up). The graded Ge content multi-layer film was observed to induce a larger normalized strain gradient.
\end{abstract}

\section{INTRODUCTION}

Polycrystalline silicon-germanium (poly-SiGe) is a promising material for surface micro-machined MEMS (MicroElectromechanical Systems) applications. Conformal deposition is possible using chemical vapor deposition techniques at temperatures below $425^{\circ} \mathrm{C}[\mathbf{1 , 2}]$. Consequently, poly-SiGe can be micro-machined directly on top of modern foundry CMOS. Moreover, research by Sedky et al. has shown that thermal annealing of CMOS devices at $525^{\circ} \mathrm{C}$ for up to 90 minutes leaves the underlying electronics largely unaffected [3].

Micromachined resonators can exhibit very high mechanical quality factors. Indeed, poly-Si MEMS resonators have been reported to have $Q$ 's in excess of 80,000 [4]. Due to their high $Q$ 's, MEMS resonators have superior frequency-selectivity compared to electronic active filters. Also, micromachined resonators are promising as replacements for discrete filters and oscillators in wireless communications systems [5,6]. Integrating RF MEMS directly with CMOS promises to drop parasitic capacitances and inductances, as well as reduce fabrication and integration costs, and the form factor of telecommunications devices.

Using low-temperature poly-SiGe, it is our aim to make the MEMS CMOS integration process straightforward and modular, particularly for RF applications. This paper lays the groundwork for this goal by characterizing single- and multi-layer poly-SiGe films with processes relevant for RF MEMS applications. Section II presents the fabrication method used to micromachine the poly-SiGe test structures. Section III summarizes $Q$ data collected from audio frequency poly-SiGe resonators. For MEMS microresonator filters and oscillators, $Q$ is of prime importance. In Section IV, the measured resonant frequencies and geometric dimensions are used to back-calculate the Young's modulus of poly-SiGe. Finally, strain gradient and the effects of RTA are discussed in Section V.

\section{FABRICATION}

All devices were fabricated using a single-mask, timedrelease process with silicon dioxide as the sacrificial layer. Single crystal silicon wafers were used as the starting substrate. Two microns of low temperature oxide (LTO) was then deposited at $400^{\circ} \mathrm{C}$. The LTO acts as both the sacrificial layer and the mechanical anchor. LTO was used as opposed to a polycrystalline germanium (poly-Ge) sacrificial layer $[\mathbf{1 , 2}]$ because of the need for electrical isolation between the mechanical anchors and the substrate.

Four structural films were studied: $1 \mu \mathrm{m}$ thick poly-SiGe films containing $62 \%, 65 \%$, or $68 \% \mathrm{Ge}$, and a $3 \mu \mathrm{m}$ tri-layer sandwich (Figure 1). All films were deposited by LPCVD at $425^{\circ} \mathrm{C}$ and in-situ boron-doped (using $\mathrm{B}_{2} \mathrm{H}_{6}$ ), yielding a deposition rate of roughly $80 \AA$ per minute. The as-deposited RMS surface roughness of the films is $50 \AA$ for the tri-layer sandwich and under $30 \AA$ for the single-layer films. The tri-layer film was used to investigate whether films with different average stresses can be combined to cancel out the residual strain gradient [7].

The resonators and strain gradient test structures used for this paper were defined using a single-mask photolithography step, followed by a standard anisotropic $\mathrm{HBr}$ plasma etch using a poly$\mathrm{Si}$ etch recipe. A timed dip in concentrated $\mathrm{HF}$ was used to release the structures while leaving $\mathrm{SiO}_{2}$ pedestals for mechanical anchoring of the test devices.

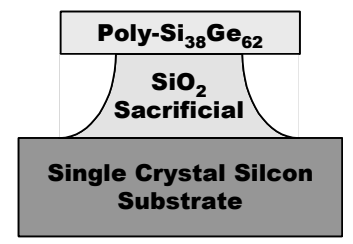

62\% Ge-content Poly-SiGe

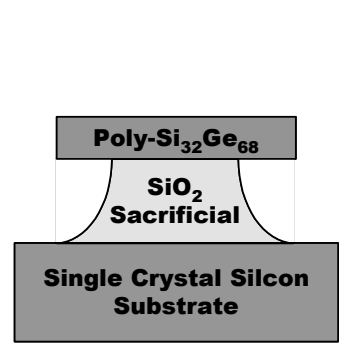

68\% Ge-content Poly-SiGe

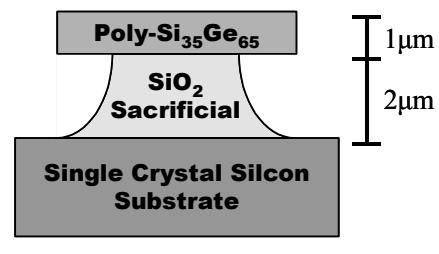

65\% Ge-content Poly-SiGe

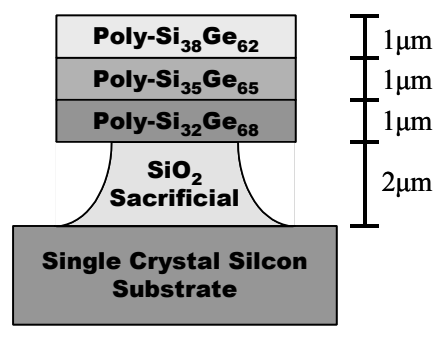

68/65/62 Tri-Layer
Figure 1. Cross-sectional views of the four types of structural films studied in this paper (depicted after HF release).

\footnotetext{
${ }^{\dagger}$ Current address: Department of Electrical Engineering, Myongji University, Kyongki, Korea.

Travel support has been generously provided by the Transducers Research Foundation and by the DARPA MEMS and DARPA BioFlips programs.
} 


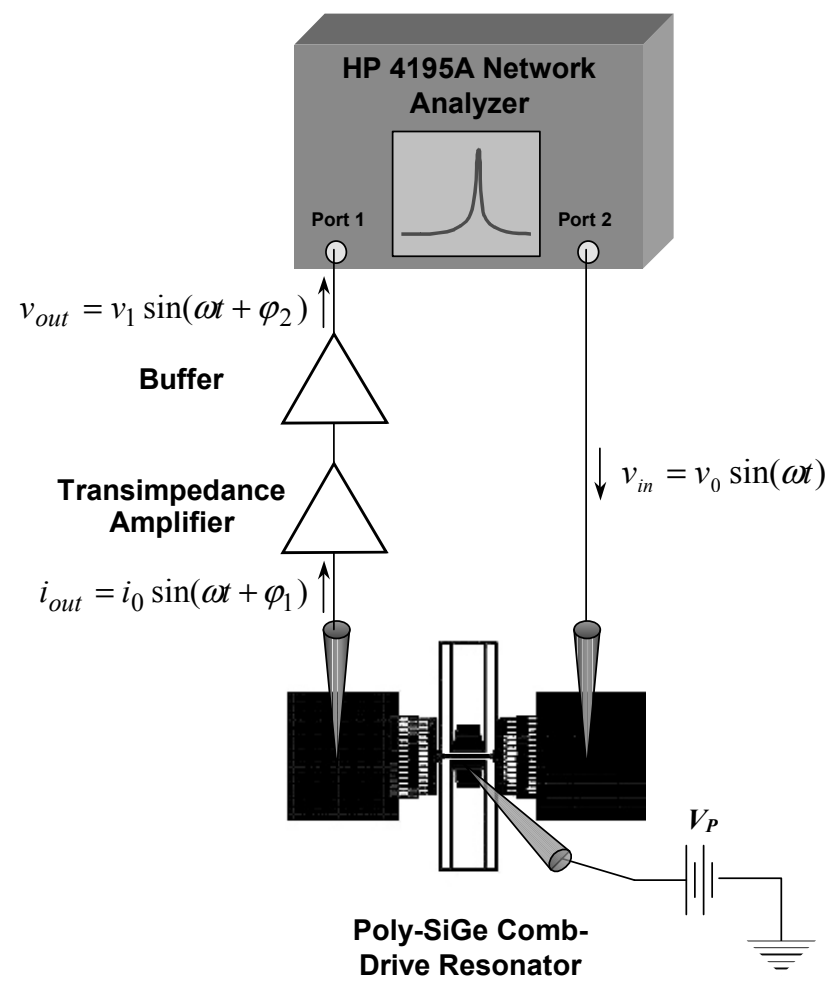

Figure 2. Schematic of the experimental test setup used for $\mathrm{Q}$ measurements. $\mathrm{V}_{\mathrm{p}}=15 \mathrm{~V}$ and $\mathrm{v}_{0}=1 \mathrm{~V}$ for the $\mathrm{Q}$ data presented in this paper. Higher offset voltages, $\mathrm{V}_{\mathrm{P}}$, result in non-linear, Duffing behavior. All tests were conducted at an ambient pressure of $2 \mu$ Torr at room temperature.

\section{III. $Q$ MEASUREMENT}

Quality factors were measured for $10.5 \mathrm{kHz}$ and $20 \mathrm{kHz}$ folded-flexure comb-drive resonators fabricated from the singleand tri-layer films mentioned above. The test setup is shown in Figure 2. Tests were conducted in an MMR vacuum probe station with conventional feedthroughs. The substrate was biased to $V_{P}$ to prevent the shuttle of the comb-drive resonators from being pulled down onto the substrate.

An HP 4195A network analyzer was used to determine the frequency response of the resonators. From the frequency response, the resonant frequency and mechanical $Q$ were determined.

As-deposited poly-SiGe has a $Q$ between 20,000 and 31,000 (Figure 3). After 1 minute RTA at $600^{\circ} \mathrm{C}$ in nitrogen ambient, the quality factor of all films increases by a factor of roughly 2.2 to 2.6. The tri-layer film had a $Q$ of 61,100 , the largest $Q$ measured to date for poly-SiGe (Figures 4 and 5). RTA reduces the defect density of the poly-SiGe films, thereby increasing the $Q$. As a result, the internal dissipation for all films decreases and, in turn, $Q$ increases. There was no discernable alteration in the resonant frequency due to RTA. Figure 6 clearly indicates that the tri-layer film consistently exhibits a higher quality factor than all of the other films. This could be due to the tri-layer resonators having larger volumes, which tends to yield higher $Q$ 's [8].

Ideally, all MEMS processing steps should be performed at CMOS compatible temperatures: deposition below $425^{\circ} \mathrm{C}$, followed by annealing up to $525^{\circ} \mathrm{C}$. Linear interpolation of Figure 3 indicates that CMOS compatible poly-SiGe has a $Q \approx 40,000-$ 44,000 , which is $1.6 \mathrm{X}-1.9 \mathrm{X}$ better than that of as-deposited films.

RTA was carried out beyond $525^{\circ} \mathrm{C}$ to establish an upper bound on $Q$. The positive linear trend of Figure 3 is evidence that the intrinsic $Q$ of poly-SiGe is higher than 61,000 . To obtain $Q$ 's greater than 44,000 and still remain CMOS compatible, longer anneal times can be used at temperatures around $525^{\circ} \mathrm{C}$.

It should be noted that determination of $Q$ was complicated by resonant-frequency drift. Accurate measurements of $Q$ take roughly 40 minutes on the HP 4195A network analyzer. We observed a downward frequency drift of roughly 1 to $4 \mathrm{~Hz}$ over this time span. Resistive heating of the resonators is negligible. Adsorption of residual oxygen, however, could be significant. Assuming the gas in the chamber is composed of $21 \% \mathrm{O}_{2}$, formation of an oxygen monolayer takes only 3 seconds at $1 \mu \mathrm{Torr}$ (assuming a densely packed monolayer and 100\% stiction rate). Therefore, absorbed gases might be causing a mass-loading affect [9], accounting for the downward drift in resonant frequency. The observed drift artificially increases or decreases the measured $Q$ depending on the sweep direction. Furthermore, for highresolution frequency measurements, the network analyzer takes multiple measurements and averages the results, which tends to smooth the resonant peak and decrease the measured $Q$. Based on the large data sets obtained, the $Q$ values presented are estimated to have an uncertainty of about $\pm 5 \%$.

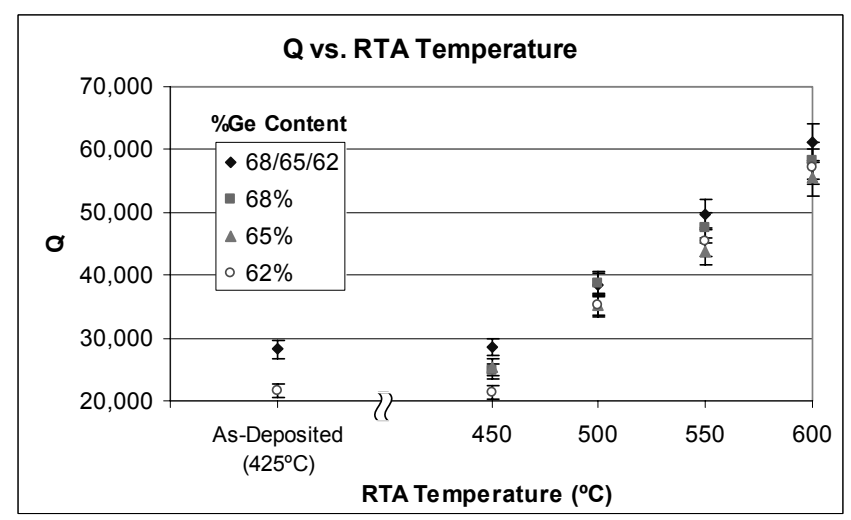

Figure 3. Graph of $\mathrm{Q}$ versus RTA Temperature. Notice that $\mathrm{Q}$ is unaltered for RTA temperatures up to approximately $450^{\circ} \mathrm{C}$. Above $450^{\circ} \mathrm{C}$, Q is roughly linear with respect to RTA temperature. All RTA was done for 1 minute at the specified temperature in nitrogen ambient.

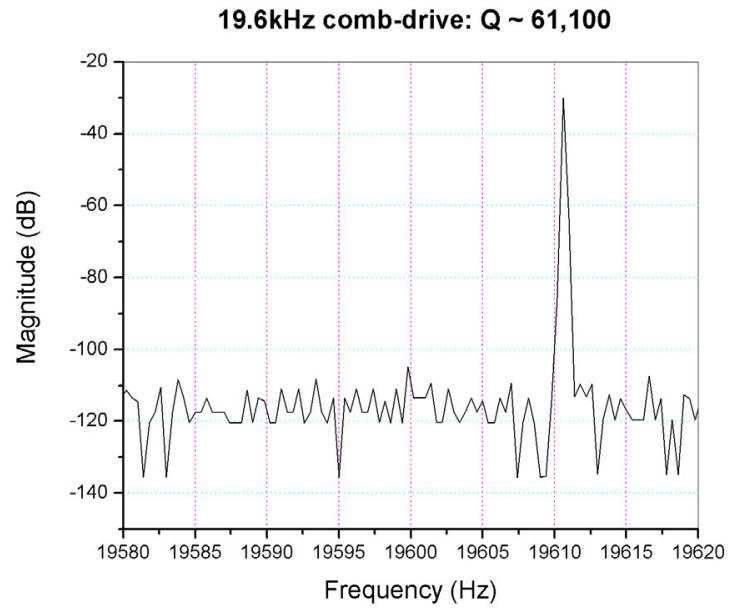

Figure 4. The magnitude (dB) versus frequency for a tri-layer poly-SiGe, $20 \mathrm{kHz}$ (design) comb-drive resonator. The peak occurs around $19.6 \mathrm{kHz}$. 


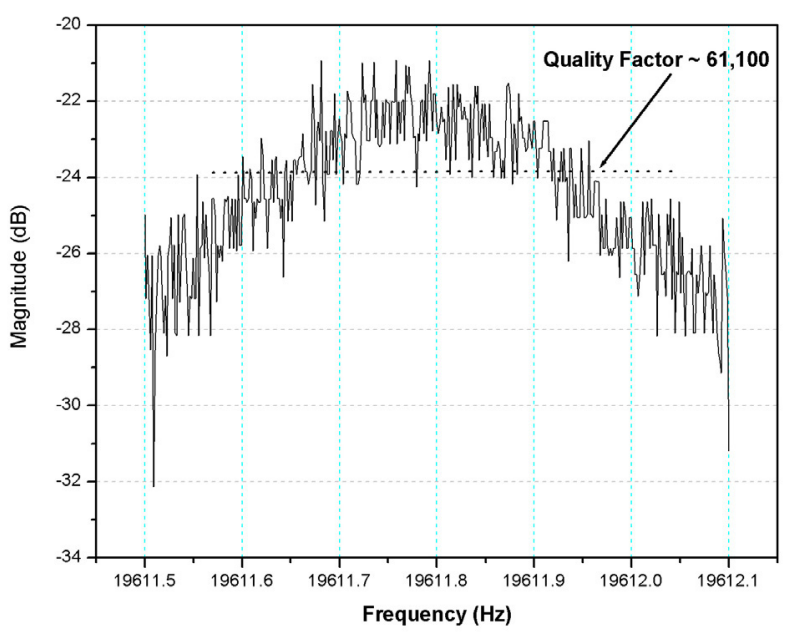

Figure 5. Close-up of the resonant peak presented in Figure 4. Q was measured from a $3 \mathrm{~Hz}$ frequency sweep near resonance.

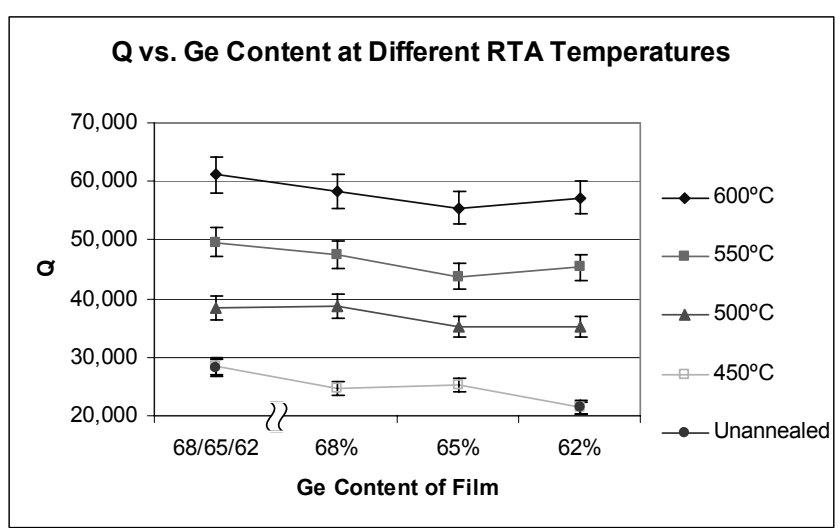

Figure 6. Graph of $\mathrm{Q}$ versus \%Ge content at different RTA Temperatures. The Q's for all films are comparable for any given set of anneal conditions.

\section{YOUNG'S MODULUS}

Young's modulus, $E$, was back-calculated from the frequency equation:

$$
f_{r}=\frac{1}{2 \pi}\left[\frac{2 E(W / L)^{3}}{\rho\left(A_{p}+0.3714 A\right)}\right]^{\frac{1}{2}}
$$

where $f_{r}$, the resonant frequency of the resonator, is estimated by Rayleigh's Energy Method [10]. $A_{p}$ and $A$ are the layout areas of the shuttle/comb fingers and the folded-flexure spring suspension, respectively. $W$ and $L$ are the width and length of the foldedflexures, and $\rho$ is the mass density of the poly-SiGe film.

Rutherford back scattering (RBS) was used to verify the mass ratio of $\mathrm{Si}$ and $\mathrm{Ge}$ in the various poly-SiGe films to within $\pm 2.0 \%$. This is an extreme upper bound, as the $\mathrm{Si} / \mathrm{Ge}$ ratio is also verified by deposition conditions. $\mathrm{A} \pm 2.0 \%$ error in the $\mathrm{Si} / \mathrm{Ge}$ ratio results in a $\pm 1.4 \%$ uncertainty in mass density $\rho$.

$W, L, A_{p}$, and $A$ were all measured to within $\pm 30 \mathrm{~nm}$ or $\pm 900 \mathrm{~nm}^{2}$ using a Wyko confocal white-light interferometer. The uncertainty in $W$ is thus approximately $\pm 1.5 \%$. Uncertainties in $L$, $A_{p}$, and $A$ are negligible. The resonant frequency, $f_{r}$, of $10.5 \mathrm{kHz}$ (design) folded-flexure resonators was determined to within $\pm 2 \mathrm{~Hz}$ with the network analyzer. Combining the measured values of $f_{r}$, $\rho, W, L, A_{p}$, and $A$ with Equation (1), $E$ was found to be $155 \mathrm{GPa}$, with a normalized uncertainty of $\pm 3.0 \%$. The metallurgical elastic modulus for a poly- $\mathrm{Si}_{35} \mathrm{Ge}_{65}$ film is $146 \mathrm{GPa}$ (i.e., the $E$ interpolated from the Young's modulii of $\mathrm{Si}$ and $\mathrm{Ge}$ based on the mass ratio of a poly- $\mathrm{Si}_{35} \mathrm{Ge}_{65}$ film). There is no discernable trend between $E$ and Ge content (Table 1).

\section{STRAIN GRADIENT}

Strain gradient was determined by measuring the out-ofplane tip deflection of $10 \mu \mathrm{m}$ wide, $300 \mu \mathrm{m}$ long cantilevers. The deflection was determined using the Wyko white-light interferometer. The strain gradient in as-deposited films decreases with increasing Ge content (Table 2). Rapid thermal annealing for one minute at $600^{\circ} \mathrm{C}$ improves the single layer strain gradients by as much as a factor of 4.7 , while the tri-layer strain gradient improves by a factor of 6.5 (Figure 7 ). The results indicate that the strain gradient is most effectively reduced by a modest anneal. Both as-deposited and annealed films curl up.

The strain gradient of the annealed tri-layer film $\left(2.67 \cdot 10^{-5}\right.$ $\left.\mu \mathrm{m}^{-1}\right)$ is 15 times higher than that of the high-temperature annealed poly-Si in Analog Devices' BiCMOS process $\left(1.78 \cdot 10^{-6} \mu^{-1}\right.$, [11]). High-frequency RF devices and, in particular, MEMS RF resonators tend to have small dimensions, rendering strain gradient of lesser importance. For such small devices, the strain gradients exhibited by the poly-SiGe films are acceptable. However, their high strain gradients make these poly-SiGe films unsuitable for many larger micromachined devices.

Table 1. Young's modulii calculated from the measured resonant frequencies of as-deposited, folded-flexure resonators with $10.5 \mathrm{kHz} \& 20 \mathrm{kHz}$ (design) resonant frequencies. The calculated Young's modulii have a normalized uncertainty of $\pm 3.0 \%$.

\begin{tabular}{|c|c|c|c|c|}
\hline $\begin{array}{c}\text { \%Ge } \\
\text { Content }\end{array}$ & $\boldsymbol{f}_{\boldsymbol{r}}(\mathbf{k H z})$ & $\boldsymbol{E}(\mathbf{G P a})$ & $\boldsymbol{f}_{\boldsymbol{r}}(\mathbf{k H z})$ & $\boldsymbol{E}(\mathbf{G P a})$ \\
\hline $68 / 65 / 62$ & 10.4 & 149 & 20.7 & 158 \\
68 & 10.6 & 156 & 20.6 & 159 \\
65 & 10.5 & 152 & 19.8 & 154 \\
62 & no data & no data & no data & no data \\
\hline
\end{tabular}

Table 2. Measured and normalized strain gradients for the various poly-SiGe films before and after RTA. RTA for 1 minute at $600^{\circ} \mathrm{C}$ reduces the curl-up of a $300 \mu \mathrm{m}$ tri-layer cantilever beam from $7.9 \mu \mathrm{m}$ to $1.2 \mu \mathrm{m}$. The tri-layer's strain gradient is, in general, lower than the single layer samples because of its greater thickness. The normalized strain gradient is simply the measured strain gradient multiplied by the film's thickness. All tip deflections were measured to within $\pm 5 \mathrm{~nm}$. Hence, all strain gradients have uncertainties less than $\pm 0.5 \%$.

\begin{tabular}{|c|c|c|c|c|}
\hline \multirow{2}{*}{$\begin{array}{c}\text { \%Ge } \\
\text { Content }\end{array}$} & \multicolumn{2}{|c|}{$\begin{array}{c}\text { Strain Gradient }\left(\boldsymbol{\mu m}^{-1}\right) \\
\text { neposited }\end{array}$} & $\begin{array}{c}\text { RTA @ } \\
\mathbf{6 0 0} \mathbf{C}\end{array}$ & $\begin{array}{c}\text { Normalized Strain } \\
\text { Gradient }\end{array}$ \\
\cline { 2 - 5 } & $\begin{array}{c}\text { As- } \\
\text { Deposited }\end{array}$ & $\begin{array}{c}\text { RTA @ } \\
\mathbf{6 0 0} \mathbf{C}\end{array}$ \\
\hline $68 / 65 / 62$ & $1.75 \cdot 10^{-4}$ & $2.67 \cdot 10^{-5}$ & $5.25 \cdot 10^{-4}$ & $8.01 \cdot 10^{-5}$ \\
68 & $2.95 \cdot 10^{-4}$ & $6.89 \cdot 10^{-5}$ & $2.95 \cdot 10^{-4}$ & $6.89 \cdot 10^{-5}$ \\
65 & $3.54 \cdot 10^{-4}$ & $7.60 \cdot 10^{-5}$ & $3.54 \cdot 10^{-4}$ & $7.60 \cdot 10^{-5}$ \\
62 & $4.68 \cdot 10^{-4}$ & $1.87 \cdot 10^{-4}$ & $4.68 \cdot 10^{-4}$ & $1.87 \cdot 10^{-4}$ \\
\hline
\end{tabular}




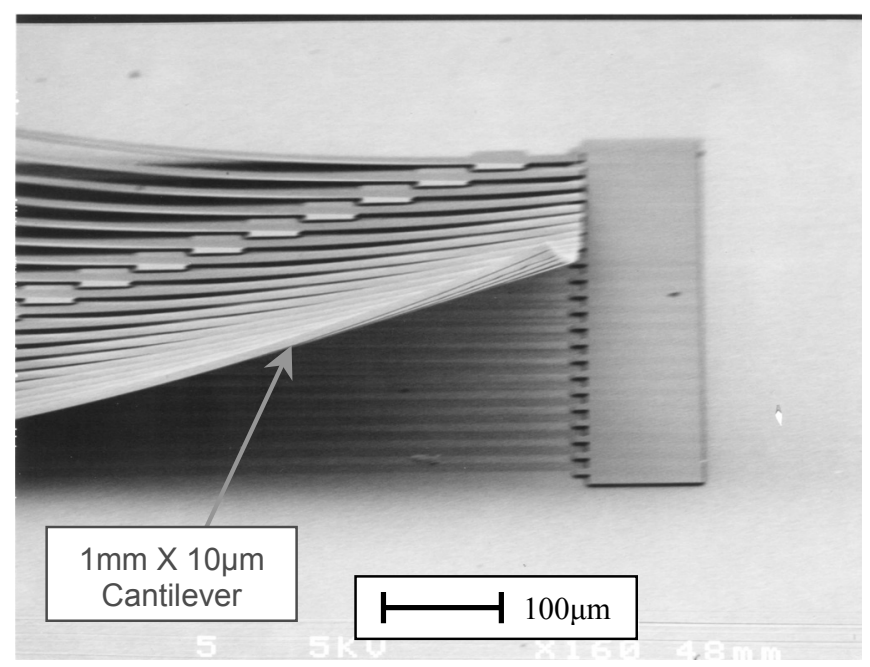

(a) As-deposited tri-layer strain gradient.

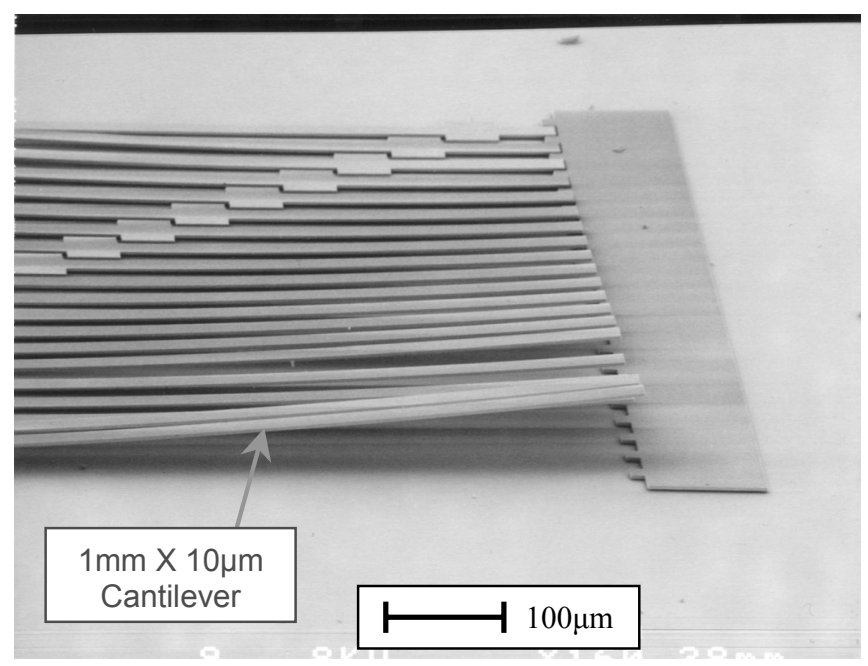

(b) Tri-layer strain gradient after one minute RTA at $600^{\circ} \mathrm{C}$.

Figure 7. Poly-SiGe strain gradient test structures asdeposited (a) and after RTA (b). As is apparent from the figure, RTA significantly reduces the strain gradient. Note: the discontinuity in tip deflections in (b) is due to the fact that one of the long cantilevers was caught under another one.

To first order, we can normalize the strain gradient by multiplying the measured strain gradient by the film's thickness. Table 3 indicates that the tri-layer film does not have improved strain gradient. Indeed, the tri-layer film exhibits higher normalized strain gradient than each of the single films. RBS data confirms that inter-layer diffusion of Ge nullified the graded $\mathrm{Ge}$ distribution in all but the top half-micron of the film, thereby canceling any positive effect that such a grading might have.

\section{CONCLUSIONS}

The $Q \approx 42,000$ and elastic modulus, $E \approx 155 \mathrm{GPa}$, of poly$\mathrm{SiGe}$ are of sufficient quality so as to make poly-SiGe attractive for integrated RF MEMS applications. For small nanoelectromechanical systems (NEMS) resonators (where large strain gradients are not overly detrimental), poly-SiGe is particularly attractive. The capability of poly-SiGe to be micromachined directly on top of modern foundry CMOS enables lower parasitics, less feedthrough, and, ultimately, lowers the cost of wireless communications devices.

The large observed strain gradient of poly-SiGe currently limits the material's application to large MEMS devices. However, the RTA results presented herein indicate that the strain gradient can be reduced significantly with modest annealing. Our group is continuing to develop methods for bringing down the strain gradient.

\section{ACKNOWLEDGEMENTS}

The authors wish to thank the DARPA MEMS program, whose generous grant (\#N66001-00-1-8955) has made this research possible. We thank Dr. Kin Man Yu at LBL for the RBS results, Marilyn Kushner for making masks at short notice, and Charley Williams for helping us fix the MMR probe station.

\section{REFERENCES}

1. A. E. Franke, Y. Jiao, M. T. Wu, T.-J. King and R. T. Howe, "Post-CMOS Modular Integration of Poly-SiGe Microstructures using Poly-Ge Sacrificial Layers," Solid-State Sensor and Actuator Workshop, Hilton Head Island, South Carolina, June 4-8 (2000), pp. 18-21.

2. A. E. Franke, "Polycrystalline Silicon-Germanium Films for Integrated Microsystems," PhD Dissertation, Dept. of Electrical Engineering and Computer Sciences, UC Berkeley (2000).

3. S. Sedky, A. Witvrouw, H. Bender and K. Baert, "Experimental Determination of the Maximum Post-Process Annealing Temperature for Standard CMOS Wafers," IEEE Transactions of Electron Devices, Vol.48 (no.2) (2001), pp. 37785 .

4. M. Biebl, G. T. Mulhern, and R. T. Howe, "In Situ Phosphorus-Doped Polysilicon for Integrated MEMS," 8th International Conference on Solid-State Sensors and Actuators and Eurosensors IX, Stockholm, Sweden (1995), pp. 198-201.

5. C. T.-C. Nguyen, "High-Q micromechanical oscillators and filters for communications," Proceedings of 1997 IEEE International Symposium on Circuits and System, Hong Kong (1997), pp. 2825-8.

6. J. R. Clark, W.-T. Hsu, and C. T.-C. Nguyen, "High-Q VHF Micromechanical Contour-Mode Disk Resonators," Technical Digest, IEEE Int. Electron Devices Meeting, San Francisco, California (2000), pp. 399-402.

7. J. Yang, H. Kahn, A-Q. He, S.M. Philips and A. H. Heuer, “A new technique for producing large-area as-deposited zero-stress LPCVD polysilicon films: the MultiPoly process," JMEMS December (2000), pp. 485-494.

8. M. L. Roukes, "Nanoelctromechanical Systems," Solid-State Sensor and Actuator Workshop, Hilton Head Island, South Carolina, June 4-8 (2000), pp. 367-376.

9. C. T.-C. Nguyen, "Micromechanical Signal Processors," $P h D$ Dissertation, Dept. of Electrical Engineering and Computer Sciences, UC Berkeley (1994), pp. 138-155.

10. W. C. Tang, C. T.-C. Nguyen, R. T. Howe, "Laterally Driven Polysilicon Resonant Microstructures," Proceedings IEEE MEMS, February (1989), pp. 53-59.

11. K. Nunan, G. Ready, and J. Sledziewski, "LPCVD \& PECVD Operations designed for iMEMS Sensor Devices," Journal of Vacuum Technology and Coating, January (2001), pp. 27-37. 